\title{
Glutamine:
}

\section{Ubiquitous and Overlooked}

\author{
By Jack M. Gorman, MD
}

Perhaps because it is virtually everywhere, glutamate has been overlooked by psychiatric researchers for a long time. The brain's major excitatory neurotransmitter, this simple amino acid is responsible for nearly half of all brain activity. Neurologists learned to understand it as the culprit in traumatic brain disease many years ago, when it was realized that much of the damage done to the brain during anoxic insults from stroke stems from overactivation of glutamate receptors, leading to increased calcium influx and neuronal death. Attempts to block this with glutamate and calcium channel antagonists have failed, but the strategy is compelling and researchers will continue to strive to make it functional in order to reduce acute brain compromise following stroke and severe head injury.

In psychiatry, focus has always been on a host of other neurotransmitters, including serotonin, noradrenaline, dopamine, and acetylcholine. Few psychiatrists remember that every time they prescribe benzodiazepines, one of the most popular classes of medication, they were stimulating another neurotransmitter, $\gamma$-aminobutyric acid, which indirectly affecting glutamate. $\gamma$-aminobutyric acid, of course, is the brain's major inhibitory neurotransmitter. It serves to brake the activity of excitatory glutamate neurons in many neural circuits. Recently, however, abnormalities in glutamate neurotransmission have risen to the forefront of attention in psychiatry and new strategies to interact with glutamate in psychiatric disease are either in the pipeline or being tested.

Perhaps the oldest story in the glutamate saga involves the theory that glutamatergic neurotransmission is deficient in schizophrenia. The most striking evidence for this comes from the work of investigators like John Krystal, MD, at Yale and Carol Tamminga, MD, now at the University of Texas, Southwestern in Dallas who showed that administration of glutamate receptor blockers, such as the anesthetic ketamine, produces a short-lived syndrome that mimics the positive, negative, and cognitive symptoms of schizophrenia. Daniel Javitt, $\mathrm{MD}, \mathrm{PhD}$, of the Albert Einstein College of Medicine in New York City, Joseph Coyle, $\mathrm{MD}$, of McLean Hospital in Belmont, Massachusetts, and others have shown that the addition of glutamate agonists to ongoing antipsychotic regimens can improve positive and negative symptoms.
Preclinically, several investigators have now shown that reducing glutamatergic neurotransmission from the prefrontal regions of the brain to the limbic areas releases dopamine from tonic inhibition, causing a subcortical hyperdominergic picture that is reminiscent of schizophrenia. Lawrence Kegeles, MD, Anissa Abi-Dargham, MD, Marc Laruelle, MD, and colleagues at Columbia University in New York City were able to show that this same relationship holds in the human brain. Hence, from a variety of perspectives, the notion that deficient glutamatergic neurotransmission may play a key role in the pathophysiology of schizophrenia is reasonable. This has led to widespread attempts to enhance glutamatergic neurotransmission as a treatment for schizophrenia.

In this issue of CNS Spectrums, Sanjay J. Mathew, MD, of Mount Sinai School of Medicine in New York City presents three stellar articles in some ways highlighting the opposite side of the schizophrenia story. Excessive glutamatergic neurotransmission may be involved in mood and anxiety disorders, leading not only to the familiar symptoms of those illnesses but also to the loss of neuronal integrity that seems to occur when they become chronic. Due to this, recent attempts to downregulate the glutamate system have become prominent in psychopharmacology research.

Because of its ubiquitous status in the brain, interacting with glutamate is not straightforward. Glutamatergic neurotransmission is required for a host of normal brain processes, including learning and memory. Total glutamatergic blockade causes seizures, dissociation, and, ultimately, death. Excessive glutamatergic stimulation causes similar devastation. Hence, the trick to modulating the glutamatergic system is subtlety. Approved drugs that cause some degree of glutamatergic downregulation include acamprosate for alcohol abuse, memantine for Alzheimer's disease, lamotrigine for bipolar disorder, and riluzole for amyotrophic lateral sclerosis.

As the articles in this issue of CNS Spectrums demonstrate, the complexity of the glutamate system also reveals the therapeutic possibilities. There appear to be many ways to subtly interact with glutamatergic neurotransmission. Discovering the right balance between excitation and inhibition could be an important key to treating mood and anxiety disorders. CNS

Dr. Gorman is the editor of this journal and professor of psychiatry and professor of neuroscience in the Department of Psychiatry at Mount Sinai School of Medicine in New York City. 